\title{
Transitions of turbulent superstructures in generalized Kolmogorov flow
}

\author{
Cristian C. Lalescu $\oplus^{1,2}$ and Michael Wilczek $\oplus^{1,3, *}$ \\ ${ }^{1}$ Max Planck Institute for Dynamics and Self-Organization, Am Faßberg 17, 37077 Göttingen, Germany \\ ${ }^{2}$ Max Planck Computing and Data Facility, Gießenbachstraße 2, 85748, Garching b. München, Germany \\ ${ }^{3}$ Institute for the Dynamics of Complex Systems, University of Göttingen, Friedrich-Hund-Platz 1, 37077 Göttingen, Germany
}

(Received 8 September 2020; accepted 23 March 2021; published 5 May 2021)

\begin{abstract}
Self-organized large-scale flow structures occur in a wide range of turbulent flows. Yet, their emergence, dynamics, and interplay with small-scale turbulence are not well understood. Here, we investigate such self-organized turbulent superstructures in three-dimensional turbulent Kolmogorov flow with largescale drag. Through extensive simulations, we uncover their low-dimensional dynamics featuring transitions between several stable and metastable large-scale structures as a function of the damping parameter. The main dissipation mechanism for the turbulent superstructures is the generation of small-scale turbulence, whose local structure depends strongly on the large-scale flow. Our results elucidate the generic emergence and low-dimensional dynamics of large-scale flow structures in fully developed turbulence and reveal a strong coupling of large- and small-scale flow features.
\end{abstract}

DOI: 10.1103/PhysRevResearch.3.L022010

Most turbulent flows, including all large-scale flows in our oceans and our atmosphere, are geometrically constrained and driven by anisotropic large-scale forces such as temperature gradients, rotation, or shear. While their small scales are typically fully turbulent, the large scales often show strikingly coherent flow patterns, termed turbulent superstructures. In boundary layer flows, turbulent superstructures appear in the form of very large-scale meandering low- and high-velocity streaks [1-3]. Convective flows, such as Rayleigh-Bénard convection, exhibit persistent large-scale convection rolls even in the fully turbulent regime [4-12]. Coherent large-scale flow states have also been observed in turbulent Taylor-Couette flow [13-16], turbulent plane Couette flow [17] as well as in von Kármán flow [18-20]. Superstructures in fully developed turbulence therefore are a widely occurring, dynamically emergent phenomenon.

Despite their generic occurrence in a broad range of prototypical flows, the emergence and dynamics of turbulent superstructures, and in particular their interplay with smallscale turbulence, are currently not well understood. However, these aspects are crucial for developing a low-dimensional description of the large-scale dynamics of fully developed turbulent flows. Moreover, Landau pointed out in a famous remark on Kolmogorov's 1941 phenomenology [21] that the large-scale structure of a flow impacts the temporal variation of the rate of energy dissipation, potentially precluding a

\footnotetext{
*michael.wilczek@ds.mpg.de
}

Published by the American Physical Society under the terms of the Creative Commons Attribution 4.0 International license. Further distribution of this work must maintain attribution to the author(s) and the published article's title, journal citation, and DOI. Open access publication funded by the Max Planck Society. universal statistical theory of turbulence valid for all flows. Therefore, a characterization of the coupling of large and small scales has far-reaching implications for the assessment of universal features (or lack thereof) of small-scale turbulence [22].

The need to simultaneously resolve the slowly evolving large scales and the rapidly fluctuating small scales renders this problem challenging, both for experiments and for simulations. To address this, we here study a prototypical shear flow-a generalized three-dimensional (3D) turbulent Kolmogorov flow, which allows the investigation of turbulent superstructures without the complications imposed by boundaries. Traditional Kolmogorov flow is a simple, twodimensional (2D) shear flow driven by a single Fourier mode, originally proposed by Kolmogorov to study the onset of turbulence on a periodic domain [23]. Indeed, the onset of turbulence [24] (for 3D, see Ref. [25]) as well as the chaotic dynamics significantly above the onset [26-28] have been studied in detail. In contrast to the simple 2D setting, the direct energy cascade toward smaller scales in three dimensions generates fully developed small-scale turbulence. Computational studies of turbulent 3D Kolmogorov flow revealed pronounced spatio-temporal large-scale intermittency and inhomogeneity [29,30], anisotropy [31], as well as translational symmetry breaking as a function of domain size [32].

Here, we generalize 3D Kolmogorov flow by including a large-scale drag term, which allows the manipulation of the range of scales on which turbulent superstructures occur. We reveal their low-dimensional dynamics in fully developed turbulence encompassing millions to billions of degrees of freedom by means of extensive simulations. In particular, we observe transitions between several large-scale structures as a function of the damping parameter. Remarkably, we find that the flow is most effectively driven for strong damping, when the large-scale flow is shaped to resonate with the shear 
forcing. The generation of small-scale turbulence acts as the main dissipation channel for the large-scale flow. A detailed spatio-temporal analysis of the energy transfer between large and small scales reveals a strong coupling of large- and smallscale flow features even in fully turbulent flows. In particular, we find that the mean rate of energy dissipation is well correlated with the energy injection rate by the large-scale forcing with a time lag associated to the energy transfer across scales. This establishes generalized Kolmogorov flow as a prototypical incarnation of a flow in which the large-scale flow variation impacts the mean rate of kinetic energy dissipation as conjectured by Landau [21].

Our Kolmogorov flow is governed by the incompressible Navier-Stokes equation

$$
\partial_{t} \boldsymbol{u}+\boldsymbol{u} \cdot \nabla \boldsymbol{u}=-\nabla p+v \Delta \boldsymbol{u}+\boldsymbol{f}-\mu \tilde{\boldsymbol{u}}
$$

on a periodic $6 \pi \times 2 \pi \times 2 \pi$ domain. Here, $\boldsymbol{u}$ denotes the incompressible velocity field $(\nabla \cdot \boldsymbol{u}=0)$, and $p$ the kinematic pressure. The shear force $\boldsymbol{f}=A \sin \left(k_{f} y\right) \boldsymbol{e}_{x}$ forces the flow on a single mode, corresponding to the smallest spanwise wave number $k_{f}=1$, with $A=1 / 2$ (code units). The linear damping term, controlled by the parameter $\mu$, only affects the sharp-Fourier-filtered field $\tilde{\boldsymbol{u}}$, which only contains spatial scales larger than the forcing scale. This permits the manipulation of large-scale flow structures through the damping parameter, while allowing for freely evolving small scales. The flow is also subject to viscous dissipation with the kinematic viscosity $v$.

To investigate the dynamics of turbulent superstructures, we conduct an extensive series of pseudospectral direct numerical simulations. We confirmed the robustness of our results for Reynolds numbers up to $R e \approx 1.8 \times 10^{4}$ (with $R e=U L / v$ based on the forcing scales $L=2 \pi / k_{f}$ and $U=(L A)^{1 / 2}$; see Supplemental Material [33]). In the following, we focus on the intermediate Reynolds number of $R e \approx 7180$.

Important features of the flow can be assessed from the 2D dynamics in the forcing plane, which we obtain by decomposing the velocity field into its $z$-averaged part and fluctuations, $\boldsymbol{u}=\boldsymbol{v}+w \boldsymbol{e}_{z}+\boldsymbol{u}^{\prime}$. Here, $\boldsymbol{v}$ denotes the $z$-averaged 2D velocity field in the forcing plane, and $w$ is the $z$-averaged vertical (out-of-plane) velocity component, which is expected to be generally small; $\boldsymbol{u}^{\prime}$ denotes the 3D velocity fluctuations. The averaged 2D dynamics follows (see also Ref. [34]):

$\partial_{t} \boldsymbol{v}+\boldsymbol{v} \cdot \nabla \boldsymbol{v}=-\nabla\langle p\rangle_{z}+v \Delta \boldsymbol{v}+\boldsymbol{f}-\mu \tilde{\boldsymbol{v}}-\nabla \cdot\left\langle\boldsymbol{u}^{\prime} \boldsymbol{u}^{\prime}\right\rangle_{z}$,

where $\langle\cdot\rangle_{z}$ denotes $z$-averaging. Apart from interactions mediated by the 3D fluctuations through the stresses, from which only horizontal (in-plane) velocity fluctuations contribute to this equation, the vertical velocity $w$ does not contribute to the dynamics of the $2 \mathrm{D}$ velocity $\boldsymbol{v}$.

From our simulations, we observe the emergence of large-scale vortex structures in the forcing plane, which we characterize by the vorticity of the $2 \mathrm{D}$ averaged velocity, $\Omega=\partial_{x} v_{y}-\partial_{y} v_{x}$. Figure 1 shows a range of different largescale states along with visualizations of the full 3D vorticity field for three different values of $\mu$. For small $\mu$, a single large-scale vortex pair emerges, which can be perceived as a strongly inhomogeneous agglomeration of small-scale vorticity. The emergence of this single vortex pair can be

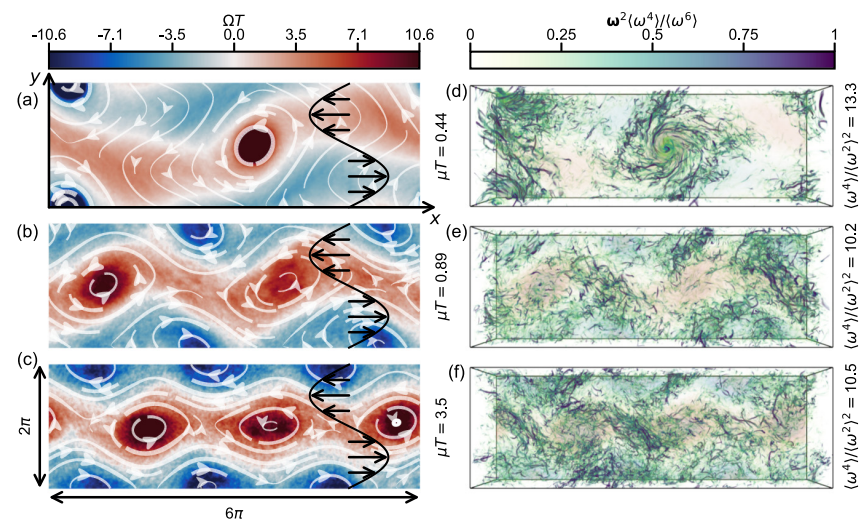

FIG. 1. Turbulent Kolmogorov flow on an aspect-ratio-three domain at $R e \approx 7180$. (a)-(c): The system exhibits different large-scale flow patterns as a function of the damping parameter $\mu$. The visualization shows the $z$-averaged vorticity $\Omega$ along with streamlines of the $2 \mathrm{D}$ flow, averaged over a time window in which the large-scale pattern is persistent (see text for details, $T=L / U$ ). (d)-(f): Volume renderings of the instantaneous vorticity field show that the spatial distribution of small-scale vortex structures and the corresponding vorticity component flatness depend on the large-scale flow state (Supplemental Videos 1 and 2).

understood as a long-wavelength instability of a flow which is initially proportional to the forcing $\left(k_{x}=0\right)$ and then destabilizes, leading to a spanwise velocity on the smallest possible wave number $\left(k_{x}=k_{f} / 3\right)$. Interestingly, this wave number is also expected based on a linear stability analysis of laminar 2D Kolmogorov flow [23,35]. By increasing $\mu$, this mode can be stabilized, triggering different flow patterns. For large $\mu$, the large-scale flow predominantly organizes into three vortex pairs. The small-scale turbulence also becomes more homogeneous, resulting in a reduced vorticity flatness when compared to the single-vortex-pair state; see Fig. 1. Since the turbulent Taylor-scale Reynolds number increases from small non-zero values of $\mu$ to larger ones, this trend is not due to an increase of small-scale turbulence [33]. Overall, this suggests that different superstructures can induce different levels of small-scale intermittency. For intermediate values of $\mu$, the flow dynamically switches between the three different states (one, two, and three vortex pairs). Therefore, the flow cannot be characterized by a single, universal large-scale flow state for a fixed set of parameters in this range. The spontaneous symmetry breaking in the form of the occurrence of one to three vortex pairs is a feature of the aspect-ratio-three domain. In domains of aspect-ratio one [29,30,32], the mean velocity field is proportional to the forcing and therefore invariant with respect to continuous $x$ translations. Larger-aspect-ratio domains, on the other hand, allow for an even greater variety of turbulent superstructures.

To characterize the large-scale dynamics, and in particular the switching between large-scale states, we compute the stream function $\psi$, given by $\Delta \psi=-\Omega$. From its Fourier transform $\hat{\psi}\left(k_{x}, k_{y}\right)$, we compute the relative intensity of the three dominant streamwise Fourier modes through $I_{j}=$ $\left|\hat{\psi}\left(j k_{f} / 3,0\right)\right|^{2} /\left[\left|\hat{\psi}\left(k_{f} / 3,0\right)\right|^{2}+\left|\hat{\psi}\left(2 k_{f} / 3,0\right)\right|^{2}+\left|\hat{\psi}\left(k_{f}, 0\right)\right|^{2}\right]$ with $j \in\{1,2,3\}$. Since $I_{1}+I_{2}+I_{3}=1$, the large-scale 


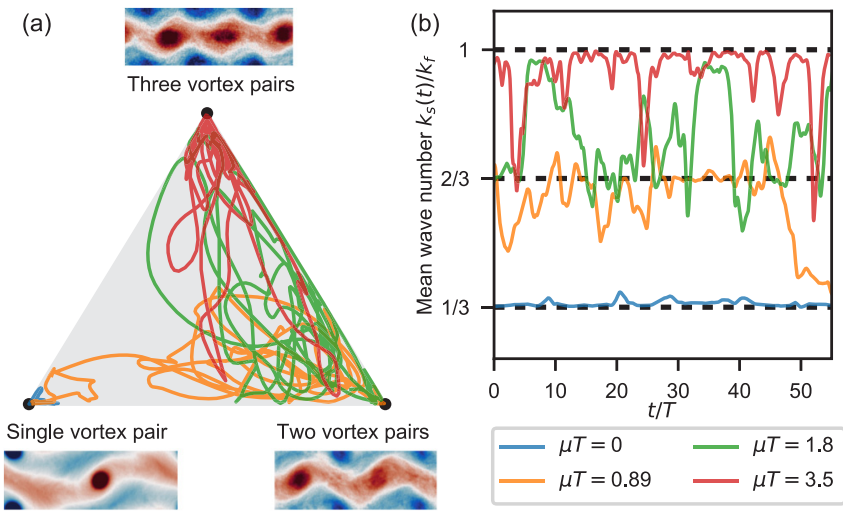

FIG. 2. The large-scale flow structures exhibit a low-dimensional dynamics. Time series of the large-scale flow states characterized by their relative intensity (a) and their mean wave number (b) show that the large-scale flow states depend on the damping parameter. For $\mu=0$, the flow organizes into a single vortex pair. For intermediate damping, the flow dynamically alternates between different largescale flow states, whereas for $\mu T=3.5$, the three-vortex-pair state is the only stable structure.

dynamics takes place in a triangle, whose corners correspond to the pure one-, two- and three-vortex-pair states. By computing a mean streamwise wave number $k_{s}=k_{f}\left(I_{1}+2 I_{2}+3 I_{3}\right) / 3$ and averaging the solution over intervals of time where $k_{s}(t)$ is approximately constant, we can identify the well-defined large-scale states shown in Fig. 1. Figure 2 shows the large-scale dynamics in the intensity plane along with the mean streamwise wave number of the large-scale structures as a function of time. For small values of the damping parameter, the large-scale flow organizes into a single vortex pair, which from time to time destabilizes due to strong, self-induced spanwise flow (see streamlines in Fig. 1) and then re-emerges (Supplemental Video 3). For intermediate damping $0.67 \leqslant \mu T \leqslant 1.8$, the large-scale flow dynamically switches between the metastable one-, two- and three-vortex-pair states (Supplemental Videos 4 and 5; Supplemental Figs. S3-S5). For large values of $\mu T \gg 1.8$, we find that only the three-vortex-pair state remains accessible to the dynamics, with bursts destroying the pattern only for it to be reformed with a possible streamwise offset (Supplemental Video 6).

To uncover the role of small-scale turbulence for the largescale dynamics, we investigate the energy budget of the flow. The total energy $E(t)=\frac{1}{2}\langle\boldsymbol{u} \cdot \boldsymbol{u}\rangle_{x y z}$ is a sum of the contributions from the $z$-averaged $2 \mathrm{D}$ velocity field $E^{2 D}(t)=$ $\frac{1}{2}\langle\boldsymbol{v} \cdot \boldsymbol{v}\rangle_{x y}$, from the $z$-averaged vertical velocity $E^{w}(t)=$ $\frac{1}{2}\left\langle w^{2}\right\rangle_{x y}$, and from the remaining 3D fluctuations $E^{\prime}(t)=$ $\frac{1}{2}\left\langle\boldsymbol{u}^{\prime} \cdot \boldsymbol{u}^{\prime}\right\rangle_{x y z}$. The energy of the 2D flow evolves according to

$$
\frac{d}{d t} E^{2 D}=\varepsilon^{f}-\varepsilon^{\mu}-\varepsilon^{\nu}-\Pi .
$$

Here, $\varepsilon^{f}(t)=\langle\boldsymbol{v} \cdot \boldsymbol{f}\rangle_{x y}$ denotes the energy input into the flow by the shear forcing, $\varepsilon^{\mu}(t)=\mu\langle\widetilde{\boldsymbol{v}} \cdot \widetilde{\boldsymbol{v}}\rangle_{x y}$ is the dissipation by large-scale damping, and $\varepsilon^{\nu}(t)=v\langle\nabla \boldsymbol{v}: \nabla \boldsymbol{v}\rangle_{x y}$ is the viscous dissipation. The term $\Pi(t)=-\left\langle\nabla \boldsymbol{v}:\left\langle\boldsymbol{u}^{\prime} \boldsymbol{u}^{\prime}\right\rangle_{z}\right\rangle_{x y}$ is the work performed by the $2 \mathrm{D}$ flow on the $3 \mathrm{D}$ stresses and characterizes
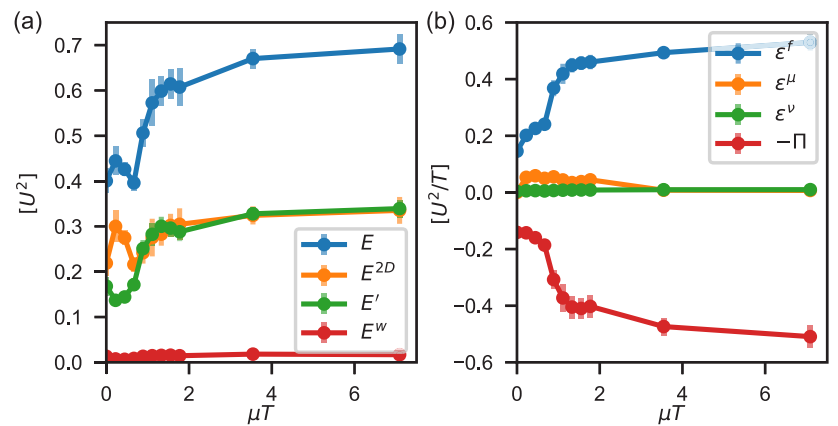

FIG. 3. Energy budget of turbulent Kolmogorov flow. (a) Different contributions to the mean total energy. The energy contained in the $2 \mathrm{D}$ flow is comparable to the energy in the 3D turbulent fluctuations. (b) Energy budget of the 2D flow. The energy input into the 2D flow $\varepsilon^{f}$ increases with increased damping. The energy injection rate is almost completely balanced by the transfer of energy $\Pi$ from the 2D component to the 3D fluctuations. Dissipation through large-scale damping and viscous diffusion are comparably negligible. Error bars span $1 \mathrm{SD}$ computed with respect to temporal variations.

the energy transfer between the $2 \mathrm{D}$ flow and the $3 \mathrm{D}$ turbulent fluctuations [36]. Figure 3(a) shows the time-averaged contributions to the kinetic energy as a function of the damping parameter $\mu$. The total energy splits up into comparable contributions from the 2D energy and the energy in 3D turbulent fluctuations, with a negligible amount of energy contained in the average vertical velocity.

Remarkably, the total energy in the flow increases as we increase $\mu$. At first, this appears counterintuitive given that we increase the strength of a dissipative term. We can explain this effect through the individual terms in the 2D budget which are shown in Fig. 3(b). The first important observation is that the energy input into the flow $\varepsilon^{f}$ increases with the damping parameter. Because $\varepsilon^{f}(t)=\langle\boldsymbol{v} \cdot \boldsymbol{f}\rangle_{x y}$, the energy input is maximal if $\boldsymbol{v} \| \boldsymbol{f}$, i.e., if the $2 \mathrm{D}$ flow is a simple shear flow in streamwise direction. Out of the three large-scale states, the three-vortex-pair state is closest to a simple shear flow, as seen by comparing the streamlines in Fig. 1 with the forcing. Tuning the flow to this state by increasing the damping therefore maximizes the energy input.

Figure 3(b) also shows that the energy input into the 2D flow is almost completely balanced by the energy transfer from the $2 \mathrm{D}$ flow to the $3 \mathrm{D}$ fluctuations. The generation of small-scale turbulence therefore is the main dissipation channel for the 2D flow. The dissipation by the large-scale damping is small for the entire parameter range, showing that its primary effect is that of shaping the large-scale flow. Because the $2 \mathrm{D}$ flow is predominantly large scale, viscous dissipation is also negligible. We have also tested the robustness of these results across Reynolds numbers (Supplemental Fig. S6).

To characterize the interplay of the large-scale flow and small-scale turbulence in more detail, we analyze how the inhomogeneity of the mean flow affects the spatial structure of the local energy transfer term $\Pi(t, x, y)=-\nabla \boldsymbol{v}:\left\langle\boldsymbol{u}^{\prime} \boldsymbol{u}^{\prime}\right\rangle_{z}$; see Fig. 4. The explicit argument $(t, x, y)$ indicates the coordinates over which no averaging was performed. The time-averaged energy transfer term is predominantly positive throughout the plane, as expected, and the large-scale state is clearly 


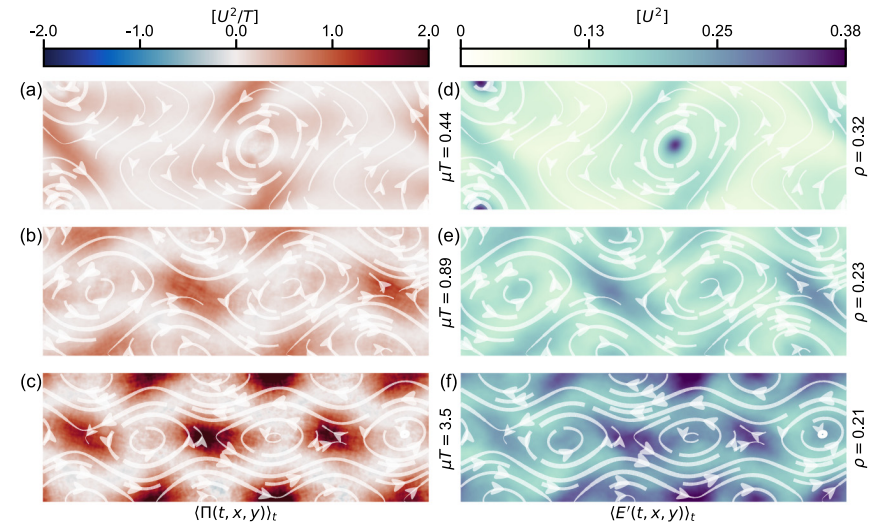

FIG. 4. The energy transfer and turbulent kinetic energy are strongly coupled to the large-scale flow (cf. Fig. 1). (a)-(c): timeaveraged energy transfer term. Energy is predominantly transferred to $3 \mathrm{D}$ fluctuations at saddle points of the averaged 2D flow (indicated by streamlines). (d)-(f): time- and $z$-averaged energy of 3D fluctuations. The energy of the fluctuations shows strong similarities to the energy transfer term. The relative standard deviation $\rho$ of the fluctuation energy as a measure of inhomogeneity depends on the large-scale state.

reflected in its spatial structure, with maxima close to saddle points of the mean flow. As this term injects energy into the 3D fluctuations, it leaves a footprint in the distribution of fluctuation energy $E^{\prime}(t, x, y)=\frac{1}{2}\left\langle\boldsymbol{u}^{\prime} \cdot \boldsymbol{u}^{\prime}\right\rangle_{z}$. Further analysis confirms that, for all three states and across all values of $\mu$, correlation coefficients of at least $60 \%$ and up to $90 \%$ are reached for the two quantities. We quantify the inhomogeneity of the 3D fluctuations by the relative standard deviation of the energy $\rho=\left\langle\left(\left\langle E^{\prime}\right\rangle_{t}-\left\langle E^{\prime}\right\rangle_{t x y}\right)^{2}\right\rangle_{x y}^{1 / 2} /\left\langle E^{\prime}\right\rangle_{t x y}$ [time average taken over time spans with the same large-scale structure, computed from $\left.E^{\prime}(t, x, y)\right]$. The values of $\rho$ are close for the two-vortex-pair and three-vortex-pair states, but significantly higher for the one-vortex-pair state. This is consistent with the vorticity flatness values given above, further highlighting the connection between the large-scale state and small-scale statistics (Supplemental Figs. S3-S5 and S7).

We now turn to the temporal evolution of the energy injection, transfer, and dissipation rates. Complementing the energy balance of $\boldsymbol{v}$, the energy budget of the spatially averaged 3D fluctuations takes the form

$$
\frac{d}{d t} E^{\prime}=\Pi-\xi^{\nu}-\xi^{w},
$$

where $\xi^{v}(t)=v\left\langle\nabla \boldsymbol{u}^{\prime}: \nabla \boldsymbol{u}^{\prime}\right\rangle_{x y z}$ denotes viscous dissipation of the 3D fluctuations and $\xi^{w}(t)=\left\langle\left\langle u_{z}^{\prime} \boldsymbol{u}^{\prime}\right\rangle_{z} \cdot \nabla w\right\rangle_{x y}$ denotes the energy transfer to the mean vertical flow. We find $\xi^{w}$ to be two orders of magnitude smaller than $\Pi$ and $\xi^{\nu}$, thus we only focus on the interplay of $\varepsilon^{f}$, $\Pi$ and $\xi^{v}$ in the following.

The temporal evolution of the self-organized large-scale flow allows us to investigate the delays between large- and small-scale flow dynamics, which are otherwise only accessible through external temporal modulation $[37,38]$. Figure 5 shows the time traces of the energy injection into the large-scale flow, the energy transfer term, and the viscous dissipation of the 3D fluctuations for a representative $\mu T=1.1$
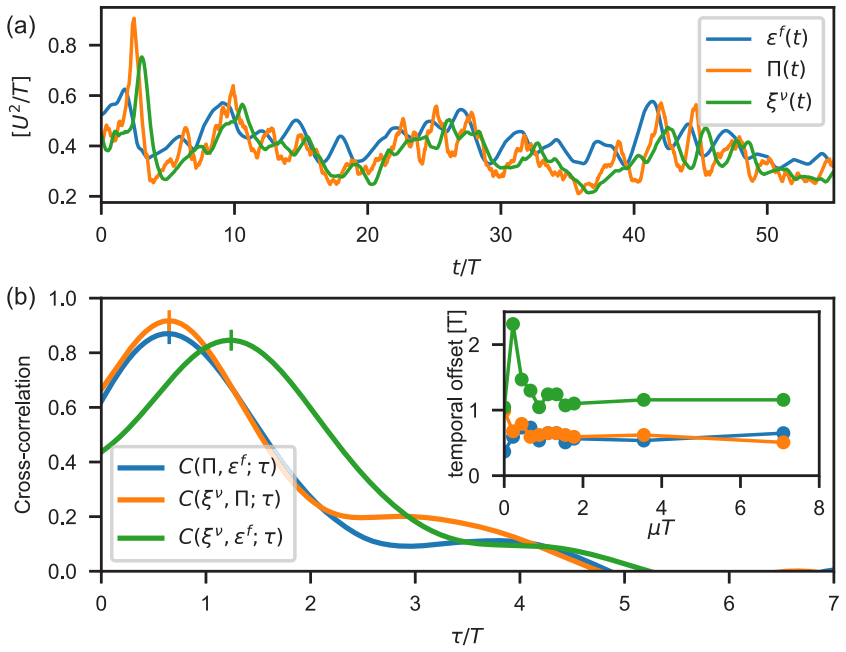

FIG. 5. Cross-correlations between large-scale forcing, energy transfer, and small-scale dissipation. Temporal evolution of energy budget terms (top) and their cross-correlations (bottom) for $R e \approx$ 7180 and $\mu T=1.1$. Cross-correlations are computed to quantify the temporal offsets between the different signals (measured as the location of the maximum correlation). The inset shows the temporal offsets for different values of $\mu$.

at $R e \approx 7180$ along with the normalized cross-correlations. Strong oscillations are evident, as well as a fairly strong correlation between the different time traces.

Cross-correlations between the energy transfer term and the large-scale energy injection, $C\left(\Pi, \varepsilon^{f} ; \tau\right)$, as well as the cross-correlations between the dissipation of 3D fluctuations and the energy transfer term, $C\left(\xi^{\nu}, \Pi ; \tau\right)$, reach values of about $90 \%$ for a temporal offset of approximately $0.6 T ; \xi^{v}(t)$ is also strongly correlated with $\varepsilon^{f}(t)$, with a temporal offset of approximately 1.2 T. The inset in Fig. 5 shows the temporal offsets between the different signals as they change with the damping parameter $\mu$. For most values of $\mu$ the offset between $\varepsilon^{f}(t)$ and $\xi^{\nu}(t)$ approximately equals the sum of the other two offsets. This can be explained by the fact that energy is transferred from the forcing scale to the dissipation scale by a direct cascade, in which the transfer between the 2D flow and the 3D fluctuations is an intermediate step. Remarkably, the two offsets are very similar in size. These observations do not hold for very small values of $\mu$, where we also find significantly lower peak correlations between the injection rate and the dissipation rate (ca. 40-50\% rather than 80-90\%).

Overall, our results draw the following picture: turbulent superstructures in Kolmogorov flow consist of accumulations of small-scale vortices, which emerge through an instability of the largest streamwise scales accessible to the dynamics. These scales can be controlled by large-scale damping. As a function of the damping parameter, we find a rich transition scenario between several large-scale states with single, two, and three vortex pairs. For intermediate damping, the system dynamically switches between various superstructures, illustrating that the system parameters do not uniquely determine the large-scale flow state. The generation of 3D turbulent fluctuations is the main dissipation channel for the large-scale flow, dominating over viscous dissipation and dissipation by 
damping. The 2D mean flow and the 3D fluctuations are strongly coupled through the energy transfer: the topology of the 2D mean flow determines the spatial distribution of the turbulent energy transfer rate, leading to inhomogeneities in the kinetic energy of the 3D fluctuations. Additional analysis shows that these inhomogeneities likely persist even at much higher Reynolds numbers. Generalized Kolmogorov flow is therefore one striking example of a flow in which the rates of energy transfer and ultimately dissipation vary considerably in response to the self-organization of large-scale superstructures, which has implications for the spatio-temporal structure of small-scale turbulence. Since a coupling of large- and small-scale flow features has been widely observed (see, e.g., Refs. $[1,3,39,40])$, the scenario outlined by Landau is presumably relevant for a large class of flows. Furthermore, our results suggest the possibility of a low-dimensional description of large-scale superstructures in fully developed turbulent flows. Since the presented mechanisms governing their emergence and dynamics are quite generic, we expect our results to be relevant for a broad range of flows including geophysical and astrophysical flows.

We thank Lukas Bentkamp, Maurizio Carbone, Gerrit Green, and Johannes Zierenberg for helpful comments on the manuscript. This work is supported by the Priority Programme SPP 1881 Turbulent Superstructures of the Deutsche Forschungsgemeinschaft. The authors gratefully acknowledge the Gauss Centre for Supercomputing e.V. for funding this project by providing computing time on the GCS Supercomputer SuperMUC at Leibniz Supercomputing Centre. Computational resources from the Max Planck Computing and Data Facility and support by the Max Planck Society are gratefully acknowledged.
[1] I. Marusic, R. Mathis, and N. Hutchins, Predictive model for wall-bounded turbulent flow, Science 329, 193 (2010).

[2] L. H. O. Hellström, B. Ganapathisubramani, and A. J. Smits, The evolution of large-scale motions in turbulent pipe flow, J. Fluid Mech. 779, 701 (2015).

[3] I. Marusic, W. J. Baars, and N. Hutchins, Scaling of the streamwise turbulence intensity in the context of inner-outer interactions in wall turbulence, Phys. Rev. Fluids 2, 100502 (2017).

[4] T. Hartlep, A. Tilgner, and F. H. Busse, Large Scale Structures in Rayleigh-Bénard Convection at High Rayleigh Numbers, Phys. Rev. Lett. 91, 064501 (2003).

[5] A. Parodi, J. von Hardenberg, G. Passoni, A. Provenzale, and E. A. Spiegel, Clustering of Plumes in Turbulent Convection, Phys. Rev. Lett. 92, 194503 (2004).

[6] T. Hartlep, A. Tilgner, and F. H. Busse, Transition to turbulent convection in a fluid layer heated from below at moderate aspect ratio, J. Fluid Mech. 544, 309 (2005).

[7] J. von Hardenberg, A. Parodi, G. Passoni, A. Provenzale, and E. A. Spiegel, Large-scale patterns in Rayleigh-Bénard convection, Phys. Lett. A 372, 2223 (2008).

[8] M. S. Emran and J. Schumacher, Large-scale mean patterns in turbulent convection, J. Fluid Mech. 776, 96 (2015).

[9] R. J. A. M. Stevens, A. Blass, X. Zhu, R. Verzicco, and D. Lohse, Turbulent thermal superstructures in Rayleigh-Bénard convection, Phys. Rev. Fluids 3, 041501(R) (2018).

[10] A. Pandey, J. D. Scheel, and J. Schumacher, Turbulent superstructures in Rayleigh-Bénard convection, Nat. Commun. 9, 2118 (2018).

[11] G. Green, D. G. Vlaykov, J. P. Mellado, and M. Wilczek, Resolved energy budget of superstructures in Rayleigh-Bénard convection, J. Fluid Mech. 887, A21 (2020).

[12] Q. Wang, R. Verzicco, D. Lohse, and O. Shishkina, Multiple States in Turbulent Large-Aspect-Ratio Thermal Convection: What Determines the Number of Convection Rolls? Phys. Rev. Lett. 125, 074501 (2020).

[13] S. G. Huisman, R. C. A. van der Veen, C. Sun, and D. Lohse, Multiple states in highly turbulent Taylor-Couette flow, Nat. Commun. 5, 3820 (2014).
[14] R. Ostilla-Mónico, D. Lohse, and R. Verzicco, Effect of roll number on the statistics of turbulent Taylor-Couette flow, Phys. Rev. Fluids 1, 054402 (2016).

[15] R. C. A. van der Veen, S. G. Huisman, O.-Y. Dung, H. L. Tang, C. Sun, and D. Lohse, Exploring the phase space of multiple states in highly turbulent Taylor-Couette flow, Phys. Rev. Fluids 1, 024401 (2016).

[16] F. Sacco, R. Verzicco, and R. Ostilla-Mónico, Dynamics and evolution of turbulent Taylor rolls, J. Fluid Mech. 870, 970 (2019).

[17] Z. Xia, Y. Shi, Q. Cai, M. Wan, and S. Chen, Multiple states in turbulent plane Couette flow with spanwise rotation, J. Fluid Mech. 837, 477 (2018).

[18] F. Ravelet, L. Marié, A. Chiffaudel, and F. Daviaud, Multistability and Memory Effect in a Highly Turbulent Flow: Experimental Evidence for a Global Bifurcation, Phys. Rev. Lett. 93, 164501 (2004).

[19] F. Ravelet, A. Chiffaudel, and F. Daviaud, Super-critical transition to turbulence in an inertially driven von Kármán closed flow, J. Fluid Mech. 601, 339 (2008).

[20] P.-P. Cortet, A. Chiffaudel, F. Daviaud, and B. Dubrulle, Experimental Evidence of a Phase Transition in a Closed Turbulent Flow, Phys. Rev. Lett. 105, 214501 (2010).

[21] L. D. Landau and E. M. Lifshitz, Fluid Mechanics, Course of Theoretical Physics, Vol. 6, 2nd ed. (Butterworth-Heinemann, Burlington, 1987).

[22] C.-C. Chien, D. B. Blum, and G. A. Voth, Effects of fluctuating energy input on the small scales in turbulence, J. Fluid Mech. 737, 527 (2013).

[23] L. Meshalkin and Y. Sinai, Investigation of stability of a steadystate solution of a system of equations for the plane motion of an incompressible viscous liquid, J. Appl. Math. Mech. (PMM) 25, 1700 (1961).

[24] A. Thess, Instabilities in two-dimensional spatially periodic flows. Part I: Kolmogorov flow, Phys. Fluids A 4, 1385 (1992).

[25] L. van Veen and S. Goto, Sub critical transition to turbulence in three-dimensional Kolmogorov flow, Fluid Dyn. Res. 48, 061425 (2016). 
[26] G. J. Chandler and R. R. Kerswell, Invariant recurrent solutions embedded in a turbulent two-dimensional Kolmogorov flow, J. Fluid Mech. 722, 554 (2013).

[27] D. Lucas and R. Kerswell, Spatiotemporal dynamics in twodimensional Kolmogorov flow over large domains, J. Fluid Mech. 750, 518 (2014).

[28] D. Lucas and R. R. Kerswell, Recurrent flow analysis in spatiotemporally chaotic 2-dimensional Kolmogorov flow, Phys. Fluids 27, 045106 (2015).

[29] V. Borue and S. A. Orszag, Numerical study of threedimensional Kolmogorov flow at high Reynolds numbers, J. Fluid Mech. 306, 293 (1996).

[30] S. Musacchio and G. Boffetta, Turbulent channel without boundaries: The periodic Kolmogorov flow, Phys. Rev. E 89, 023004 (2014).

[31] K. P. Iyer, F. Bonaccorso, L. Biferale, and F. Toschi, Multiscale anisotropic fluctuations in sheared turbulence with multiple states, Phys. Rev. Fluids 2, 052602(R) (2017).

[32] I. E. Sarris, H. Jeanmart, D. Carati, and G. Winckelmans, Box-size dependence and breaking of translational invariance in three-dimensional turbulent Kolmogorov flows, Phys. Fluids 19, 095101 (2007).
[33] See Supplemental Material at http://link.aps.org/supplemental/ 10.1103/PhysRevResearch.3.L022010 for an overview of the simulations, a description of the Supplemental Videos, as well as a characterization of the Reynolds number dependence of our results.

[34] A. Alexakis and L. Biferale, Cascades and transitions in turbulent flows, Phys. Rep. 767-769, 1 (2018).

[35] S. Chatterjee and M. K. Verma, Kolmogorov flow: Linear stability and energy transfers in a minimal low-dimensional model, Chaos 30, 073110 (2020).

[36] G. L. Eyink, Locality of turbulent cascades, Physica D 207, 91 (2005).

[37] D. Lohse, Periodically kicked turbulence, Phys. Rev. E 62, 4946 (2000).

[38] A. von der Heydt, S. Grossmann, and D. Lohse, Response maxima in modulated turbulence, Phys. Rev. E 67, 046308 (2003).

[39] O. R. H. Buxton and B. Ganapathisubramani, Concurrent scale interactions in the far-field of a turbulent mixing layer, Phys. Fluids 26, 125106 (2014).

[40] P. Berghout, W. J. Baars, and D. Krug, The large-scale footprint in small-scale Rayleigh-Bénard turbulence, J. Fluid Mech. 911, A62 (2021). 